\title{
Evolutionary Dilemmas in a Social Network
}

\author{
L. Luthi* $\quad$ E. Pestelacci ${ }^{*} \quad$ M. Tomassini ${ }^{\ddagger}$
}

\begin{abstract}
We simulate the prisoner's dilemma and hawk-dove games on a real social acquaintance network. Using a discrete analogue of replicator dynamics, we show that surprisingly high levels of cooperation can be achieved, contrary to what happens in unstructured mixing populations. Moreover, we empirically show that cooperation in this network is stable with respect to invasion by defectors.
\end{abstract}

\section{Introduction}

Some extremely simple games lead to puzzles and dilemmas that have a deep social meaning. The Prisoner's Dilemma (PD), a universal metaphor for the tension that exists between social welfare and individual selfishness, is the most famous game of this type. It stipulates that, in situations where individuals may either cooperate or behave selfishly and thus defect, they will rationally choose the latter. Unfortunately, cooperation would be the preferred outcome when global welfare is considered. Game theory [14] is the discipline that deals with such situations of conflict where two or more individuals must make decisions that will mutually influence each other. It takes a view of collective systems in which global social outcomes emerge as a result of the interaction of the individual decisions made by each agent. Another well known simplified model of many common important socioeconomic situations is the Hawk-Dove (HD) game. According to game theory, cooperative attitude should vanish in the $\mathrm{PD}$, and should be limited to a given fraction in the HD. This is also the case when large populations of individuals play the game pairwise in a random manner and anonymously, as prescribed by evolutionary game theory [16. However, in controlled experiments it has been observed that cooperation actually emerges when the

\footnotetext{
*Information Systems Department, University of Lausanne, Switzerland

${ }^{\dagger}$ Information Systems Department, University of Lausanne, Switzerland

${ }^{\ddagger}$ Information Systems Department, University of Lausanne, Switzerland
} 
game is played by humans and in many other cases [3, 9]. A number of mechanisms have been invoked to explain the emergence of cooperative behavior: repeated interaction, reputation, and belonging to a recognizable group have often been mentioned [3]. However, the work of Nowak and May [8] showed that simply arranging the players in a spatial structure and allowing them to only interact with neighbors is sufficient to sustain a certain amount of cooperation even when the game is played anonymously and without repetition. Nowak and May's study and much of the following work was based on regular structures such as two-dimensional grids (see also [4] for the HD case). However, while two-dimensional grids may be realistic for ecological and some biological applications, they are inadequate for modeling human networks of interactions as it has now become clear that many actual networks have a structure that is neither regular nor random but rather of the small-world type. Roughly speaking, small-world networks are graphs in which any node is relatively close to any other node. In this sense, they are similar to random graphs but unlike regular lattices. However, in contrast with random graphs, they also have a certain amount of local structure, as measured, for instance, by a quantity called the clustering coefficient which essentially represents the probability that two neighbors of a given node are themselves connected (see e.g. [6]). Thus, most real conflicting situations in economy and sociology are not well described neither by a fixed geographical position of the players in a regular lattice nor by a mixing population, and it becomes relevant to study these dilemmas on other, more faithful social structures. Some previous work has been done in this direction. We mention Santos and Pacheco's work on scale-free networks [10, 11] and work on Watts-Strogatz small-world graphs [1, 12, 15. However, these network types, although they have the right global "statistical" properties, are only an approximation of the actual topological properties of measured networks of interactions. In the present work we introduce a more socially relevant network and we emphasize the relationships between community structure and cooperation. A recent work close to the present one in spirit is Holme et al. [5]. However, the authors of [5] only study the PD on a much smaller social network using a different, noisy, strategy update rule, while we employ the more standard replicator dynamics on a larger social network and also study the HD game.

The remainder of this paper is organized as follows. We first give a brief background on the PD and HD. We then describe the main features of social networks and we present an evolutionary game model on a real collaboration network. We finally present and discuss results of numerical simulations of the model in terms of cooperation, community structure and stability. 


\section{The Model}

\subsection{Social Dilemmas}

We first recall a few elementary notions on the PD and the HD games. These are two-person, symmetric games in which each player has two possible strategies: cooperate (C) or defect (D). In strategic form, these games have the payoff bi-matrix shown in table 1. In this matrix, $\mathrm{R}$ stands for the reward the two players receive if they both cooperate, $\mathrm{P}$ is the punishment for bilateral defection, and $\mathrm{T}$ is the temptation, i.e. the payoff that a player receives if it defects, while the other cooperates. In this latter case, the cooperator gets the sucker's payoff S. For the PD, the payoff values are ordered numerically in the following way: $T>R>P>S$, while in the HD game $T>R>S>P$. Defection is always the best rational individual choice in the $\mathrm{PD}-(\mathrm{D}, \mathrm{D})$ is the unique Nash equilibrium and also an evolutionary stable strategy (ESS). Mutual cooperation would be preferable but it is a strongly dominated strategy. Thus the dilemma is caused by the "selfishness" of the actors. In the HD, when both players defect

\begin{tabular}{c|cc} 
& $\mathrm{C}$ & $\mathrm{D}$ \\
\hline $\mathrm{C}$ & $(\mathrm{R}, \mathrm{R})$ & $(\mathrm{S}, \mathrm{T})$ \\
$\mathrm{D}$ & $(\mathrm{T}, \mathrm{S})$ & $(\mathrm{P}, \mathrm{P})$
\end{tabular}

Table 1: Payoff matrix for $2 \times 2$ symmetric games.

they each get the lowest payoff; $(\mathrm{C}, \mathrm{D})$ and $(\mathrm{D}, \mathrm{C})$ are Nash equilibria of the game in pure strategies, and there is a third equilibrium in mixed strategies where strategy $\mathrm{D}$ is played with probability $1 /(2 \beta-1)$, and strategy $\mathrm{C}$ with probability $1-1 /(2 \beta-1)$, where $\beta$ is another name for the temptation $T$. The dilemma in this game is caused by "greed", i.e. players have a strong incentive to "bully" their opponent by playing D, which is harmful for both if the outcome is $(\mathrm{D}, \mathrm{D})$.

\subsection{Social Networks}

In standard evolutionary game theory [14, 16, these dilemmas have been classically studied by modeling the behavior of a large population in which randomly paired individuals play the game in an anonymous manner. Nonrational players are "hard-wired" to play a given strategy, and those faring better than average increase their share in the population. The fixed points of these replicator dynamics are evolutionarily stable strategies, i.e. they cannot be invaded by a mutant strategy [16]. 
In terms of networks of interaction, the "mixing" structure of the population would be represented by a complete graph, i.e. any individual may interact with any other player. The advantage of the mixing model is that it admits an approach by mean-field methods, which treat the system as being homogeneous, ignoring space dependences and correlations [16]. However, we know today that real social networks do not have this random structure. Instead, they are of finite size, have heterogeneous connectivity, are often small worlds, in the sense that any individual is only a few steps away from any other, and individuals cluster together in communities [2, 6, 15]. Therefore, evolutionary games should be studied on more general types of graphs, to understand the limitations of the theory, and to extend it as far as possible to structures encountered in real-life.

As stated in the introduction, numerical simulations of evolutionary games have been performed for degree-homogeneous and degree-heterogeneous model graphs such as Watts-Strogatz and scale-free networks [1, 10, 11, 12, 15. Here we go a step further and make use of a real social collaboration network, the genetic programming coauthorship network. This network is a small world with a connected giant component of 942 scientists and it has recently been analyzed 13 . It has clusters and communities and it should be representative of other similar human acquaintance networks. WattsStrogatz networks [15] are only a mathematical construction and do not properly represent social networks. As for model scale-free graphs, most social networks studied to date are not of the pure scale-free type, and show a faster decay of the tail of the degree distribution [2, 6]. Intuitively, there must be a cutoff in the number of acquaintances a given agent can have, and in many cases also a typical number of acquaintances, which gives a scale to the network. Besides, it has been observed that social networks have a higher clustering than the typical values reached in scale-free graphs, another manifestation of the complex neighborhood structure of the network. Furthermore, the appearance of communities - sets of densely connected vertices with sparse connections between the sets - is yet another typical feature found in social structures. Communities can highly influence the way information is propagated throughout the network or opinion formation is processed. Finally, we should make it clear that social networks are dynamical, i.e. new nodes may join the network forming new links, and old nodes may leave it as social actors come and go. As a first approximation here we model a static network, thus ignoring fluctuations and non-equilibrium phenomena. 


\subsection{Model Parameters}

Population Structure. We consider a population $P$ of players of size $N$. Each individual $i$ in the population $P$ is represented as a vertex $v_{i}$ of a graph $G(V, E)$, with $v_{i} \in V, \forall i \in P$. An interaction between two players $i$ and $j$ is represented by the undirected edge $e_{i j} \in E$. The number of neighbors $N(i)$ of player $i$ is the degree $k_{i}$ of vertex $v_{i}$. The average degree of the network will be called $\bar{k}$. The terms vertex, node, individual, or player shall be used interchangeably in the sequel; likewise for edge, link, interaction, and acquaintance.

Strategy Update Rules. To update the strategies of the individuals given an initial strategy distribution in the population, we use a discrete analogue of replicator dynamics [4]. Other socially meaningful strategy update policies could also be used, such as imitation of the best and proportional updating [4, 8, 12]. The replicator dynamics assumes that the share of the population playing a particular strategy grows in proportion to how well this strategy is doing relative to the average population payoff.

Let $\Pi_{x}$ be a player $x$ 's aggregated payoff and $k_{x}$ the number of neighbors $x$ has $\left(k_{x}\right.$ can also be seen as the degree of the vertex representing $x$ ). We define the replicator dynamics function $\phi\left(\Pi_{j}-\Pi_{i}\right)$ as being the probability function according to which player $i$ adopts neighbor $j$ 's strategy, namely

$$
\phi\left(\Pi_{j}-\Pi_{i}\right)= \begin{cases}\frac{\Pi_{j}-\Pi_{i}}{k_{j} \Pi_{M 1}-k_{i} \Pi_{m 1}} & \text { if } \Pi_{j}-\Pi_{i}>0 \\ 0 & \text { otherwise }\end{cases}
$$

where $\Pi_{M 1}$ (resp. $\Pi_{m 1}$ ) is the maximum (resp. minimum) payoff a player could get if it had only one neighbor.

Payoff Calculation. There exist several possibilities for determining a player's utility or payoff. One can define a player's payoff as being the sum (accumulated payoff) of all pair interactions with its nearest neighbors. Or it can be defined as the accumulated payoff divided by the number of interactions (average payoff). Accumulated and average payoff give the same results when considering degree-homogenous networks such as regular lattices. Accumulated payoff seems more logical to use in degree-heterogeneous networks since it reflects the very fact that players may have different numbers of neighbors in the network. Average payoff, on the other hand, smoothes 
out the possible differences although it might be justified in terms of the number of interactions that a player may sustain in a given time, i.e. an individual with many connections is likely to interact less often with each of its neighbors than another that has a lower number of connections. Also, if there is a cost to maintain a relationship, average payoff will roughly capture this fact, while it will be hidden if one uses accumulated payoff. In this paper we use a form of accumulated payoff.

Population Dynamics. Calling $C(t)=\left(s_{1}(t), s_{2}(t), \ldots, s_{N}(t)\right)$ a configuration of the population strategies $s_{i} \in\{C, D\}$ at time step $t$, the global synchronous system dynamics leads to $C(t+1)$ by simultaneously updating all the players' strategies according to the chosen rule. Synchronous update, with its idealization of a global clock, is customary in spatial evolutionary games, and most results have been obtained using this model. However, perfect synchronicity is only an abstraction as agents normally act at different and possibly uncorrelated moments. In spite of this, it has been shown that the update mode does not fundamentally alter the results for replicator dynamics [4]. We have also checked that asynchronous update dynamics does not influence the system evolution in a significant way and so, all results presented refer to synchronous systems.

\section{Simulation Results and Analysis}

For each game, we can explore the entire game space by limiting our study to the variation of only two parameters per game. In the case of the PD, we set $R=1$ and $S=0$, and vary $1 \leq T \leq 2$ and $0 \leq P \leq 1$. For the HD game, we set $R=1$ and $P=0$ and the two parameters are $1 \leq T \leq 2$ and $0 \leq S \leq 1$. In the Prisoner's Dilemma case, $P$ is limited between $R=1$ and $S=0$ in order to respect the ordering of the payoffs $(T>R>P>S)$ and $T$ 's upper bound is equal to 2 due to the $2 R>T+S$ constraint. Had we instead fixed $R=1$ and $P=0, T$ could be as big as desired, provided $S \leq 0$ is small enough. In the Hawk-Dove game, setting $R=1$ and $P=0$ determines the range of $S$ (since this time $T>R>S>P$ ) and gives an upper bound of 2 for $T$, again due to the $2 R>T+S$ constraint. Note however, that the only valid value pairs of $(T, S)$ are those that satisfy the latter constraint.

The network is randomly initialized with $50 \%$ cooperators and $50 \%$ defectors. In all cases, the parameters are varied between their two bounds by steps of 0.1 . For each set of values, we carry out 50 runs of 16000 time steps 
each. Cooperation level is averaged over the last 1000 time steps, well after the transient equilibration period.

\subsection{Evolution of Cooperation}

In Figure 1 we report average cooperation levels for both games for systems having attained a steady-state. As expected, the region in which cooperation is possible is much more restricted in the $\mathrm{PD}$ than for HD. Cooperation is more widespread for the HD, as mutual defection is the worst outcome in this game. For the PD cooperation is sensitive to the "punishment" level $\mathrm{P}$, for a given $\mathrm{T}$. Concerning the $\mathrm{HD}$, one can see that the $\mathrm{S}$ parameter has moderate influence on cooperation for a given $\mathrm{T}$. We also notice that the transition from cooperation to defection is much steeper in the PD than for the HD.

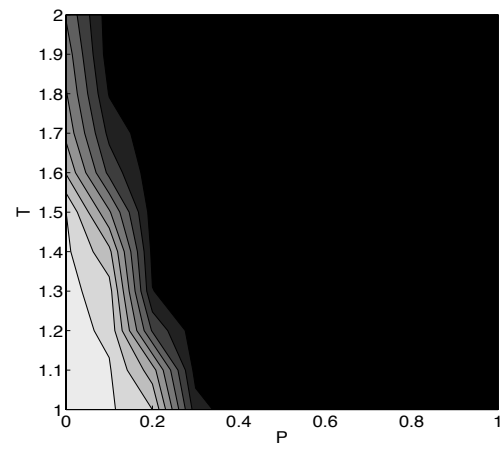

(a)

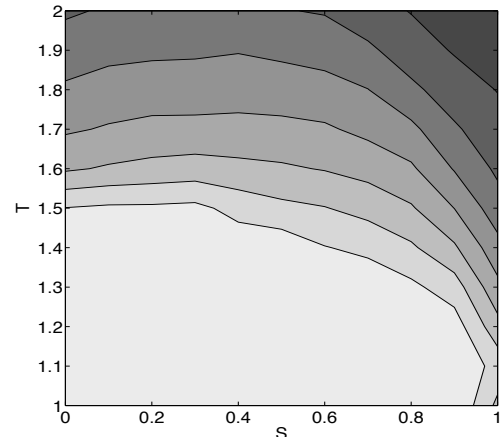

(b)

Figure 1: Level of cooperation at the end of the simulation. Left: PD; right: HD. For the HD, the meaningful phase space is the lower left triangle only.

Another important global quantity is the total payoff at the end of the simulated games, also called the wealth. The cumulated wealth of defectors and cooperators is plotted in Figure 2 for the PD. This is done for $T=1.3$, for two values of the punishment $\mathrm{P}$, giving rise to two different cooperation regimes: one in which cooperation prevails and a second one where defection predominates. We see that the cooperators' wealth is larger and has a broader distribution. This hints at a clustering of cooperators, as this is the only way for them to increase their payoff. We shall comment on this phenomenon below.

Social networks are characterized by the presence of communities, which 


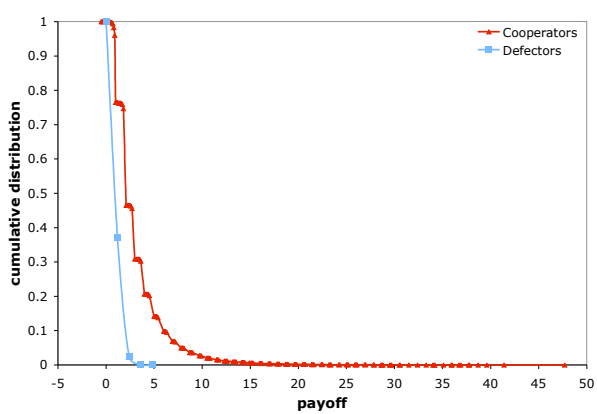

(a)

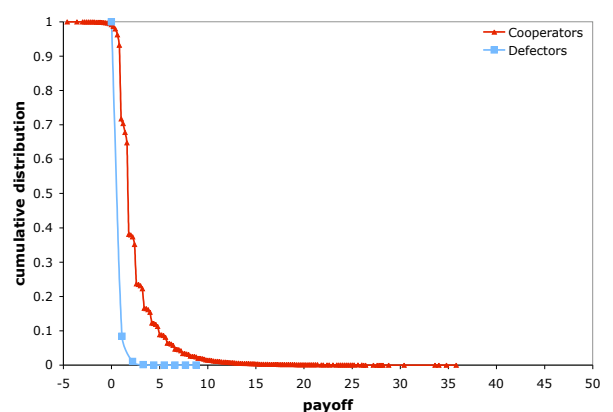

(b)

Figure 2: Cumulative wealth distribution in the PD game averaged over 50 runs for the social network model; (a) $T=1.3, P=0.1$ yielding $\sim 73 \%$ of cooperation. Average $\mathrm{C}$-wealth $=2.92$, average $\mathrm{D}$-wealth $=0.47$; (b) $T=$ $1.3, P=0.2$ yielding $\sim 15 \%$ of cooperation. Average $\mathrm{C}$-wealth $=2.30$, average $\mathrm{D}$-wealth $=0.11$.

can be seen as sets of highly connected vertices having few connections with vertices belonging to other communities. Finding the communities in a given network is a difficult task for which there exist several algorithms essentially based on clustering considerations. We have used one of the algorithms proposed by Newman [7].

For reasons of space, in the following we show results for the PD only. In Figure 3 we depict a portion of the scientific coauthorship graph, distinguishing between cooperators and defectors for the PD. We note that tightly-bound communities are mostly composed of players with the same strategy. Although we only show a small portion of the whole network for reasons of clarity, we could have chosen many other places as the phenomenon is widespread. Cooperators tend to "protect" themselves by having many links toward other cooperators. On the other hand, a cooperator like the central one in the largest defecting community will have a tendency to become a defector since its neighbors are nearly all defectors; but when its highly connected "wealthy" cooperator neighbor on the left of the figure is probabilistically selected to be imitated, then it will certainly become a cooperator again. So, the rare cooperators that are not tightly clustered with other cooperators will tend to oscillate between strategies. The community structure of cooperators, together with the mutual payoff advantage of cooperating, explains the previous observation, namely that the average cooperators' wealth exceeds the average wealth of defectors. Strategy 
distribution in communities found for the HD game is qualitatively similar; however, in the HD the two strategies are slightly more intermingled, confirming analogous findings for grid-structured populations [4].

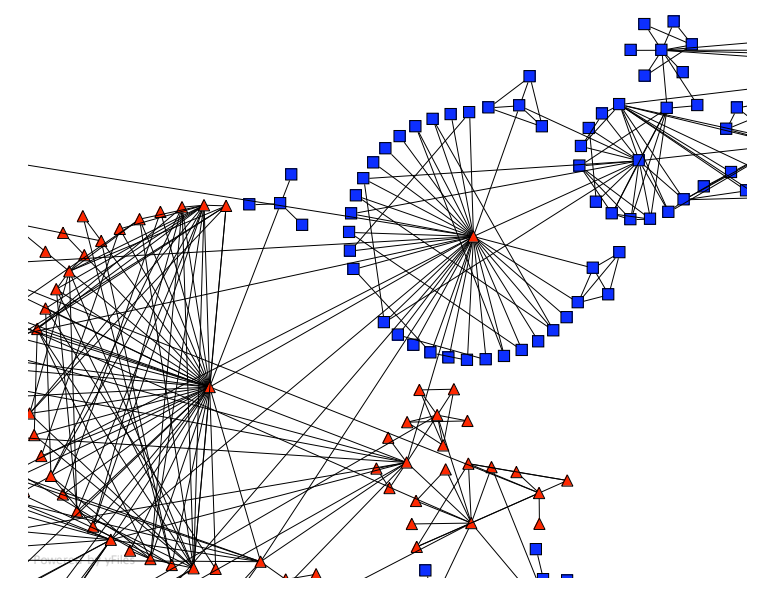

Figure 3: Communities: cooperators are represented by triangles and defectors by squares.

When cooperation prevails, we have found that cooperators tend to occupy the highest degree nodes. When defectors predominate, the degree distributions of the two strategies tend to be closer, although the cooperators still monopolize higher degree nodes. For HD the results are similar, namely, degree distribution for defectors fall off more rapidly than those for cooperators.

\subsection{Evolutionary Stability}

No empirical investigation of an evolutionary games scenario would be complete without examining its evolutionary stability [16]. Evolutionary stability can be defined exactly for mixing populations but qualitatively it simply implies that a given population strategy cannot be invaded by an individual playing another strategy. For example, a single defector in a mixing population of cooperators in the PD will lead to a total extinction of the cooperators. Single individual strategy mutations are interesting only in mixing populations or in networks of the scale-free type [11. In our social network, the distribution of strategies that obtains when the steady-state is reached is left undisturbed by this kind of event. However, given that the $\mathrm{C}$ and $\mathrm{D}$ strategies tend to cluster together, we have applied a more 
radical type of perturbations to the system. After the pseudo-equilibrium is reached, we choose a highly connected individual that plays the strategy of the majority (suppose it is C) and we flip its strategy to D as well as the strategy of all its first neighbors that are also cooperators. In this case, evolutionary stability requires that any such small group of individuals who try an alternative strategy do worse than those that those who stick to the status quo. Figure 4 shows the results using the above described perturbation. In each figure, ten executions have been reported to give a feeling of the behavior (many more have been run but in the case of noise, average values are irrelevant). Figures 4 (a), (b), and (c) refer to the PD with $P=0.1$ and

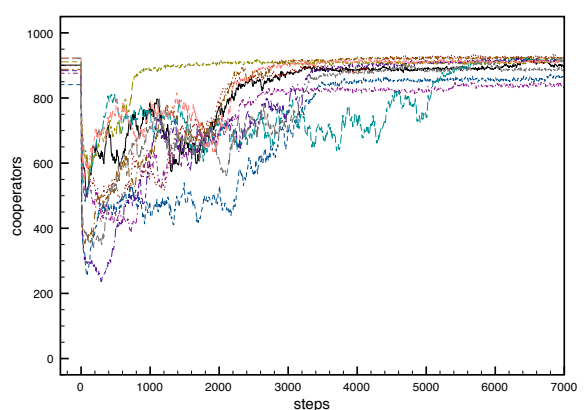

(a)

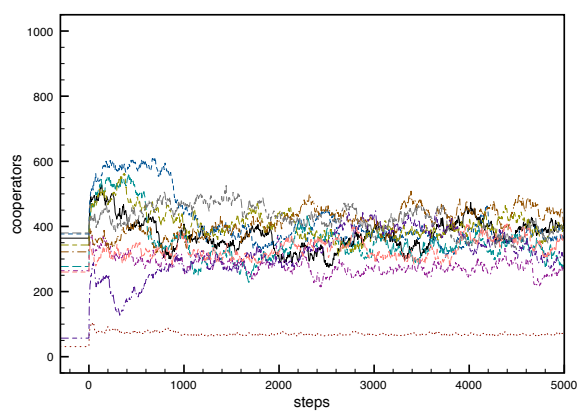

(c)

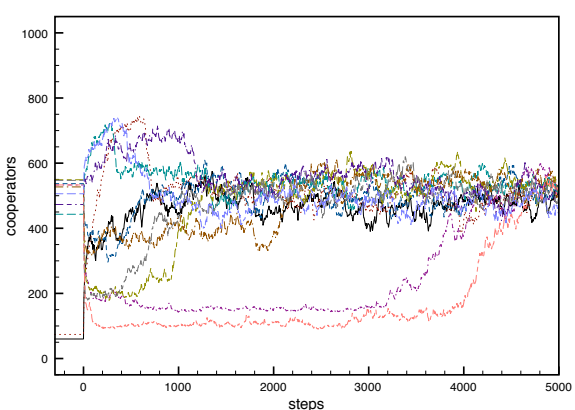

(b)

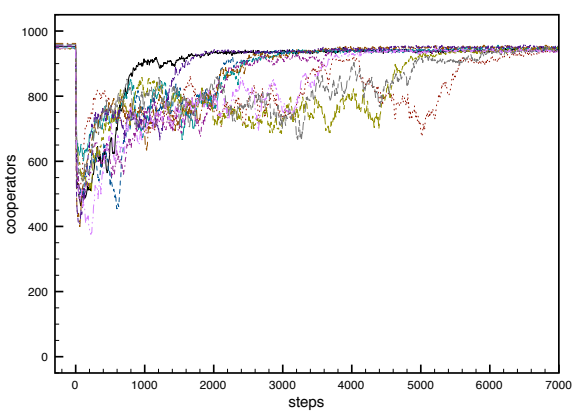

(d)

Figure 4: Effect of noisy behavior on pseudo-equilibria states for the PD (a), (b), (c), and HD (d) (see text for explanations).

three different values of $T: 1.5,1.7$, and 1.9 respectively. Figure 4 (d) refers to the HD with $S=0.1, T=1.6$. The first constant part of the graphs up to abscissa 0 represent the pseudo-equilibrium values reached in each run after 10000 steps, just before applying the perturbation. For the PD, it can 
be seen that where cooperation is high (figure 4 (a)), after a transient period in which there can be a significant loss of cooperation, all runs tend to recover the original levels, albeit at different speeds. When cooperation is at intermediate or lower levels (figures 4 (b) and (c)), the behavior is more oscillatory but populations tend to recover the original pseudo-equilibrium levels of cooperation. Even populations that had originally a significantly lower cooperation percentage can sometimes reach the cooperation level of the majority of runs after the perturbation. For the HD, populations easily recover from noisy behavior (see figure $4(\mathrm{~d})$ ), as cooperation is more widespread in this game. Even for extreme values of $T$ the original cooperation level tends to be recovered (not shown to save space but very similar to figure 4 (b)).

\section{Conclusions}

Extending previous work on regular structures and on model scale-free and small-world networks, in this paper we have empirically studied two fundamental social dilemmas on a real acquaintance network. Although the graph studied is a single particular instance, it possesses all the features that characterize actual social networks, such as high clustering and communities. We find that this kind of topology allows cooperation to be reached and maintained, for a large portion of the game parameter space for $\mathrm{HD}$, and even in the more difficult case of the PD. It was previously known that this is the case for lattice structures and, most notably, for scale-free graphs. However, these structures are not good representations of social ties and thus our result is encouraging from the social point of view. Importantly, we have also shown that the quasi-equilibria reached by the dynamics are not ephemeral, unstable states; on the contrary, they are very robust against perturbations represented by strategy flips of groups of agents. When reshuffled by the perturbation, even population configurations in which defection prevails either recover the previous level of cooperation or increase it, which means that this kind of social graphs intrinsically favor cooperation through clustering and tight communities. In the future, we would like to generalize these results to classes of social network models.

Acknowledgments. Financial support for this research by the Swiss National Science Foundation under contract 200021-107419 is gratefully acknowledged. 


\section{References}

[1] G. Abramson and M. Kuperman. Social games in a social network. Phys. Rev. E, 63:030901, 2001.

[2] L. A. N. Amaral, A. Scala, M. Barthélemy, and H. E. Stanley. Classes of small-world networks. Proc. Natl. Acad. Sci. USA, 97(21):11149-11152, 2000 .

[3] R. Axelrod. The Evolution of Cooperation. Basic Books, Inc., New York, 1984.

[4] C. Hauert and M. Doebeli. Spatial structure often inhibits the evolution of cooperation in the snowdrift game. Nature, 428:643-646, April 2004.

[5] P. Holme, A. Trusina, B. J. Kim, and P. Minhagen. Prisoner's dilemma in real-world acquaintance networks: spice and quasi-equilibria induced by the interplay between structure and dynamics. Phys. Rev. E, 68:030901(R), 2003.

[6] M. E. J. Newman. The structure and function of complex networks. SIAM Review, 45:167-256, 2003.

[7] M. E. J. Newman. Fast algorithm for detecting community structure in networks. Phys. Rev. E, 69:066133, 2004.

[8] M. A. Nowak and R. M. May. Evolutionary games and spatial chaos. Nature, 359:826-829, October 1992.

[9] D. Sally. Conversation and cooperation in social dilemmas: A metaanalysis of experiments from 1958 to 1992. Rationality and Society, $7: 58-92,1995$.

[10] F. C. Santos and J. M. Pacheco. Scale-free networks provide a unifying framework for the emergence of cooperation. Phys. Rev. Lett., 95:098104, 2005.

[11] F. C. Santos, J. M. Pacheco, and T. Lenaerts. Evolutionary dynamics of social dilemmas in structured hetrogeneous populations. Proc. Natl. Acad. Sci. USA, 103:3490-3494, 2006.

[12] M. Tomassini, L. Luthi, and M. Giacobini. Hawks and doves on smallworld networks. Phys. Rev. E, 73:016132, 2006. 
[13] M. Tomassini, L. Luthi, M. Giacobini, and W. B. Langdon. The structure of the genetic programming collaboration network. Genetic Programming and Evolvable Machines, 8(1):97-103, 2007.

[14] F. Vega-Redondo. Economics and the Theory of Games. Cambridge University Press, Cambridge, UK, 2003.

[15] D. J. Watts. Small worlds: The Dynamics of Networks between Order and Randomness. Princeton University Press, Princeton NJ, 1999.

[16] J. W. Weibull. Evolutionary Game Theory. MIT Press, Boston, MA, 1995. 